\title{
Les sanctuaires ruraux gallo-romains en territoire arverne et vellave
}

\section{Claire Mitton}

\section{(2) OpenEdition \\ Journals}

Édition électronique

URL : http://journals.openedition.org/adlfi/4756

ISSN : 2114-0502

Éditeur

Ministère de la Culture

Référence électronique

Claire Mitton, «Les sanctuaires ruraux gallo-romains en territoire arverne et vellave », ADLFI.

Archéologie de la France - Informations [En ligne], Auvergne, mis en ligne le 01 mars 2006, consulté le 14 novembre 2019. URL : http://journals.openedition.org/adlfi/4756

Ce document a été généré automatiquement le 14 novembre 2019.

(c) Ministère de la Culture et de la Communication, CNRS 


\title{
Les sanctuaires ruraux gallo- romains en territoire arverne et vellave
}

\author{
Claire Mitton
}

Identifiant de l'opération archéologique : 118

Date de l'opération : 2006 (PT)

1 Cette étude, qui est la première synthèse sur le thème des sanctuaires en Auvergne, a permis de recenser toutes les mentions de sites cultuels. Un indice de fiabilité ayant été attribué à chacune de ces mentions, les sites ont pu être classés et les sanctuaires attestés ont été regroupés au sein d'une typologie dans laquelle toutes les données ont été analysées et comparées.

2 Un dépouillement bibliographique et photographique exhaustif a été effectué. Cela a permis de recenser cent quatre-vingt-neuf mentions de lieux de culte pour la région Auvergne dont cent cinquante-cinq concernant les peuples arvernes et vellaves.

3 Quarante-six de ces mentions correspondent à des sanctuaires attestés et onze correspondent à des sanctuaires hypothétiques. Quarante et un sites possèdent un indice de fiabilité de deux sur quatre, c'est-à-dire que pour ces mentions, plusieurs théories sont viables, dont celles d'un lieu de culte. Enfin, neuf sites possèdent un indice de fiabilité de un et quarante-trois sites un indice de zéro : ce sont des mentions liées à la tradition, sans aucun fondement, et des sites dont la qualité de lieu de culte a été ôtée après réinterprétation.

4 Parmi les quarante-six occurrences attestant un site cultuel et possédant un indice de fiabilité quatre, trente sites sont utilisables au sein de la typologie. Les autres correspondent à des sites sans aménagements ou à des sites ne comportant aucun plan connu. Parmi ces trente sites, six d'entre eux comportent plusieurs structures identifiées à des fanaou à des temples, ainsi il y a un total de trente-six temples 
enregistrés dans ce classement. Précisons que toutes les structures sont arvernes, car aucun plan vellave n'est connu.

À propos des dimensions, on remarque que les fanaà une cellamesurent en majorité entre $10 \mathrm{~m}$ et $12 \mathrm{~m}$ et n'excèdent pas $17 \mathrm{~m}$ de côté, sauf pour deux exemples : Landeyrat et La Chapelle-Marcousse. Ces deux sites se remarquent par leur monumentalité : ils mesurent $50 \mathrm{~m}$ de côté. Concernant les temples hybrides, on constate qu'ils se mesurent tous entre $15 \mathrm{~m}$ et $25 \mathrm{~m}$. Quant aux fanaà doublecelloe, leur longueur est évidemment plus grande, car ils reçoivent la présence de deux structures. Les dimensions sont d'environ $25 \mathrm{~m}$ de côté, sauf pour un site : celui de Ruynes-enMargeride (Cantal, [023]) qui se distingue, car il mesure $50 \mathrm{~m}$ de côté. Le même schéma se profile dans les études générales portant sur la Gaule, où la longueur moyenne des galeries des sanctuaires est située entre $10 \mathrm{~m}$ et $17 \mathrm{~m}$. Les galeries au-delà de $20 \mathrm{~m}$ appartiennent à des fanaà double cellceou polygonaux, ou encore à des temples urbains (voir Fauduet Isabelle, 1993, Les temples de tradition celtique en Gaule romaine,Paris, 160 p.).

On remarque que les trois sites qui se distinguent par leurs dimensions importantes atteignant $50 \mathrm{~m}$ de côté sont situés en zone de montagne : Landeyrat (Cantal, [014]) et Ruynes-en-Margeride (Cantal, [023]) sont localisés dans le Cantal, à respectivement $1050 \mathrm{~m}$ et $900 \mathrm{~m}$ d'altitude; La Chapelle-Marcousse (Puy-deDôme, [019]) est dans le Puy-de-Dôme, sur le versant septentrional du plateau du Cézallier, à $1163 \mathrm{~m}$ d'altitude. L'étude a également porté sur les lieux de culte sans aménagement. Il se définit, par cet intitulé, des sanctuaires qui ne présentent pas de bâtiment construit dédié au culte : il s'agit de lieux honorés pour eux-mêmes, le plus généralement divinisés en raison de leur cadre naturel propice (source, grotte, bois). Les puits cultuels sont également intégrés dans cette partie, car leurs éléments de construction ne relèvent généralement pas d'un caractère cultuel. La majorité des puits ne possède pas de fonction votive à la base, la nature domestique est souvent détournée pour en faire un lieu de culte. Une analyse spatiale a également été effectuée. Ainsi, on constate par exemple que les zones d'altitude ne sont pas un frein à l'implantation des sanctuaires. Des temples ont été découverts jusqu'à $1300 \mathrm{~m}$ d'altitude, et même $1465 \mathrm{~m}$ avec celui situé au sommet du puy de Dôme. On remarque que dix sanctuaires sur un total de quarante-six sites attestés ont une altitude supérieure ou égale à $1000 \mathrm{~m}$ et sept sites ont une altitude entre $700 \mathrm{~m}$ et $1000 \mathrm{~m}$. N'oublions pas également que si moins de sites sont découverts en altitude, cela s'explique simplement par le fait que la recherche est beaucoup moins intense dans ces zones que dans la plaine (notamment la 
plaine de la Limagne qui a concentré les recherches). Les sanctuaires peuvent également avoir un lien avec d'autres marqueurs du territoire, tels que les voies de communication. Une voie antique est visible sur des photographies tout à côté du sanctuaire de Charbonnier-les-Mines ou de celui de La Sauvetat. De même, les lieux de culte peuvent être situés à proximité de villa(comme à Aigueperse) ou de thermes (comme au Mont-Dore). On remarque également des regroupements de sanctuaires également en zone rurale comme à Saint-Julien-de-Coppel où deux sanctuaires et un temple sont situés à moins de $200 \mathrm{~m}$ les uns des autres. Soit ils appartiennent à un même complexe religieux, soit ils se succèdent dans le temps. Les structures étant contemporaines, une spécialisation des sanctuaires est également envisageable, mais difficilement prouvable. Il serait intéressant d'étudier l'environnement archéologique de ces trois sites pour tenter de voir dans quel contexte ils s'insèrent: ce vaste ensemble cultuel est peut-être à mettre en relation avec une agglomération. Cette hypothèse est toutefois pour l'instant difficilement envisageable en l'absence de données plus précises.

17 MITTON Claire

\section{INDEX}

Index géographique : Auvergne

peuple Arverne, Vellaves

Index chronologique : Empire romain, Protohistoire

operation Prospection thématique (PRT)

Thèmes : analyse des données, analyse documentaire, analyse spatiale, cella, communauté rurale, culte, fanum, galerie, lieu sacré, pronaos, puits, sanctuaire, temple, thermes, typologie, villa, voie romaine

\section{AUTEURS}

CLAIRE MITTON

BEN 